\title{
Nonlinear vibration analysis of functionally graded carbon nanotube reinforced fluid-conveying tube in thermal environment
}

\author{
Xu Chen ${ }^{\#}$, Jing-Lei Zhao ${ }^{\#}$, Gui-Lin She, Yan Jing, Hua-Yan Pu* and Jun Luo \\ College of Mechanical and vehicle Engineering, Chongqing University, Chongqing,400044, China
}

\section{1 abstract}

In this paper, the nonlinear free vibration responses of functionally graded nanocomposite fluid-conveying tube reinforced by single-walled carbon nanotubes (SWNTs) in thermal environment is investigated. The SWCNTs gradient distributed in the thickness direction of the tube forms different reinforcement patterns. The materials properties of the functionally graded carbon nanotube-reinforced composites (FGCNTRC) are estimated by rule of mixture. A higher-order shear deformation theory and Hamilton's variational principle are employed to derive the motion equations incorporating the thermal and fluid effects. A two-step perturbation method is implemented to obtain the closed-form asymptotic solution for these nonlinear partial differential equations. The nonlinear frequency under several patterns of reinforcement are presented and discussed. We conducted a series of studies aimed at revealing the effects of the flow velocity, environment temperature, geometrical ratios $R_{i} / R_{o}$ and carbon nanotube volume fraction on the nature frequency.

Keywords: nonlinear vibration; fluid-conveying tube; carbon nanotube; thermal load; two-step perturbation method;

\subsection{Introduction}

Due to carbon nanotubes have excellent mechanical properties, i.e. high specific strength, high specific modulus and low density ${ }^{[1-5]}$. Since they were discovered by Iijima $^{[6]}$, they have been considered as excellent reinforcement of composite materials. Studies by many scholars show that, the mechanical ${ }^{[7]}$, electrical ${ }^{[8]}$ and thermal properties $^{[9]}$ of the polymer can be significantly improved by mixing carbon nanotubes

\footnotetext{
${ }^{*}$ Corresponding author, Professor.

E-mail: phygood 2001@shu.edu.cn

\# These authors contributed equally
} 
with the polymer matrix at a certain mass fraction. Functionally graded materials (FGM) was first proposed by Japanese researchers in the 1980s. The principle is that reinforced materials are distributed inhomogeneous in space, which can change the mechanical properties of beams, plates and shells. Shen ${ }^{[10]}$ first applied the concept of functionally graded materials to SWNTS reinforced nanocomposite plates, allowing the SWNTS to be graded distributed along the desired direction in an isotropic matrix. Since then, many scholars including Shen have studied FG-CNTRC beam, tube, plate, shell and other structures ${ }^{[10-26]}$. Readers can refer to the comprehensive literature review prepared by Liew ${ }^{[27]}$ for relevant researches.

Tube is the basic unit of many engineering structures, which is widely used in oil, chemical and nuclear industry. Therefore, many researchers have used different analytical and numerical methods to study the stability and vibration analysis of fluid conveying tube. Zhen ${ }^{[28]}$ first studied the nonlinear vibration of the supercritical fluidconveying tube composed of FGM with initial curvature. Liang ${ }^{[29]}$ investigate the stability and nonlinear parametric vibration of a spinning fluid-conveying tube using analytical and numerical methods. Rahim Abdollahi ${ }^{[30]}$ studies the stability of a spinning fluid-conveying tube under simultaneous internal and external fluid loads using Euler-Bernoulli beam theory. Zhu ${ }^{[31]}$ studies the free and forced vibration of a fluid-conveying tube composed of FGM under elastic support based on Euler-Bernoulli beam theory and considering von Karman assumption and damping effect. Zhou ${ }^{[32]}$ explore the nonlinear vibrations of cantilever fluid-conveying tube under axial excitation. Tan ${ }^{[33]}$ studies forced vibration of fluid-conveying tube under supercritical condition by using Timoshenko beam theory. Based on Euler-Bernoulli beam theory, Shahali ${ }^{[34]}$ studies the nonlinear dynamic response of fluid-conveying tube under the action of uniform external cross flow. A.R. Askarian ${ }^{[35]}$ studies the vibration response of fluid-conveying tube under general boundary conditions using Euler-Bernoulli beam theory. $\mathrm{Lu}^{[36]}$ first studies the influence of vibration on fatigue performance of FGM fluid-conveying tube based on Euler-Bernoulli beam theory. Khodabakhsh ${ }^{[37]}$ studies $^{3}$ the post-buckling and nonlinear vibration of FGM fluid-conveying tube based on the Timoshenko beam theory. M. Heshmati ${ }^{[38]}$ studies the stability and free vibration of FGM fluid-conveying tube with eccentric geometric defects.

As far as we know, there are no researches on nonlinear vibration of functionally graded carbon nanotube reinforced fluid-conveying tube in thermal environment by using higher-order shear deformation theory. In this paper, an investigation on the 
nonlinear free vibration responses of functionally graded nanocomposite fluidconveying tube reinforced by SWNTs in thermal environment is presented. A higherorder shear deformation theory and Hamilton's variational principle are employed to derive the governing equations incorporating the thermal and fluid effects. A two-step perturbation method is implemented to obtain the closed-form asymptotic solution for these nonlinear partial differential equations. The nonlinear frequency under several patterns of reinforcement are presented and discussed. We conducted a series of studies aimed at revealing the effects of the flow velocity, environment temperature, geometrical ratios $R_{i} / R_{o}$ and carbon nanotube volume fraction on the nature frequency.

\subsection{Material properties of FG-CNTRC tube}

Consider a fluid-conveying FG-CNTRC tube shown in Fig. 1. The tube, having length $\mathrm{L}$, inner radius $\mathrm{Ri}$ and outer radius Ro, are simply supported at both ends. It is assumed that the tube is subjected to a transverse load $\mathrm{q}$ and uniform thermal loading. The reference coordinate system is also depicted in Fig.1. It is clear that $y=r \cos \theta, z=r \sin \theta$.

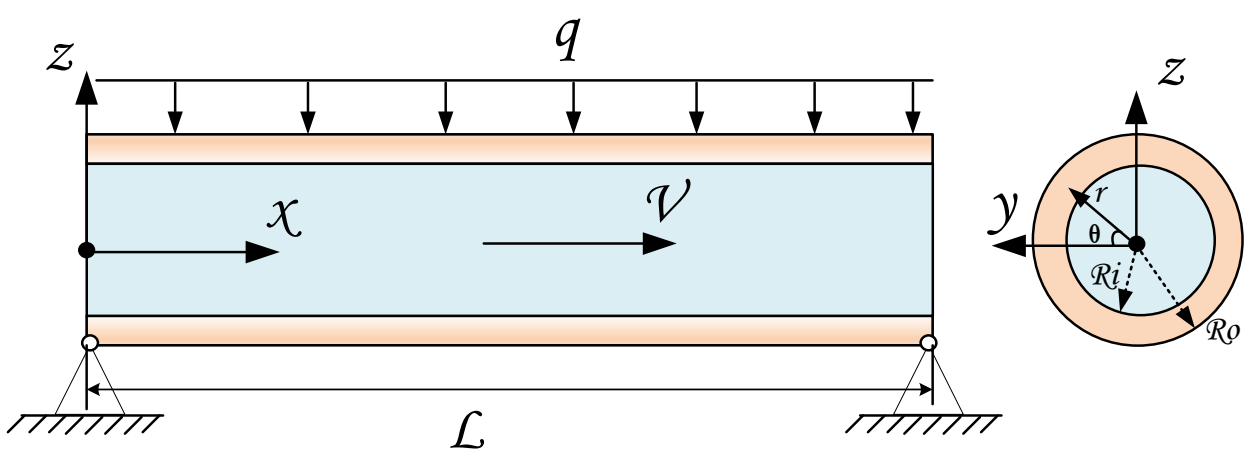

Fig. 1 Schematic diagram of a nanocomposite fluid-conveying tube with simply support.

The effective material properties of FG-CNTRC beams can be estimated by the rule of mixture ${ }^{[39]}$.

$$
\begin{gathered}
E_{11}=\eta_{1} V^{C N T} E_{11}^{C N T}+V^{m} E^{m} \\
\frac{\eta_{2}}{E_{22}}=\frac{V^{C N T}}{E_{22}^{C N T}}+\frac{V^{m}}{E^{m}}
\end{gathered}
$$

With $E_{11}^{C N T}, E_{22}^{C N T}, G_{12}^{C N T}$ being the Young's modulus and shear modulus of the SWCNT, respectively, and $G^{m}, E^{m}$ being the corresponding material properties of the matrix. $V^{C N T}, V^{m}$ are the volume fractions of the carbon nanotube and the matrix, with the relationship of $V^{C N T}+V^{m}=1$. Carbon nanotube efficiency parameters $\eta_{i}(i=1,2,3)$ are introduced to characterize the interaction between matrix and CNT and load transfer. 
They can be determined by the Molecular dynamic simulations and the rule of mixture model. Following the rule of mixture, Poisson's ratio and mass density of the FGCNTRC tube are as follows.

$$
\begin{aligned}
& v_{12}(r)=v_{12}^{C N T} V^{C N T}+v^{m} V^{m} \\
& \rho(r)=\rho^{C N T} V^{C N T}+\rho^{m} V^{m}
\end{aligned}
$$

In which, $v_{12}^{C N T}, v^{m}$ and $\rho^{C N T}, \rho^{m}$ are defined as the Poisson's ratios and mass density of the CNT and matrix, respectively.

In this paper, five types of the FG-CNTRC tube were considered, which have the form as:

$$
\begin{gathered}
V^{C N T}(r)=V_{r e}^{C N T} \\
V^{C N T}(r)=2\left(\frac{r-R_{i}}{R_{o}-R_{i}}\right) V_{r e}^{C N T} \\
V^{C N T}(r)=-2\left(\frac{r-R_{i}}{R_{o}-R_{i}}\right) V_{r e}^{C N T} \\
V^{C N T}(r)=2\left(1-2 \cdot \frac{\left.\mid \begin{array}{l}
\mid \\
R_{o}-\frac{R_{o}+R_{i}}{2} \mid
\end{array}\right)}{\left|r-\frac{R_{o}+R_{i}}{2}\right|} V_{r e}^{C N T}\right. \\
V^{C N T}(r)=4 \cdot \frac{\mid R_{o}-R_{i}}{C N T}
\end{gathered}
$$

Where, equations (5)-(9) represent UD-beam, V-beam, $\wedge$-beam, O-beam and Xbeam, respectively. 


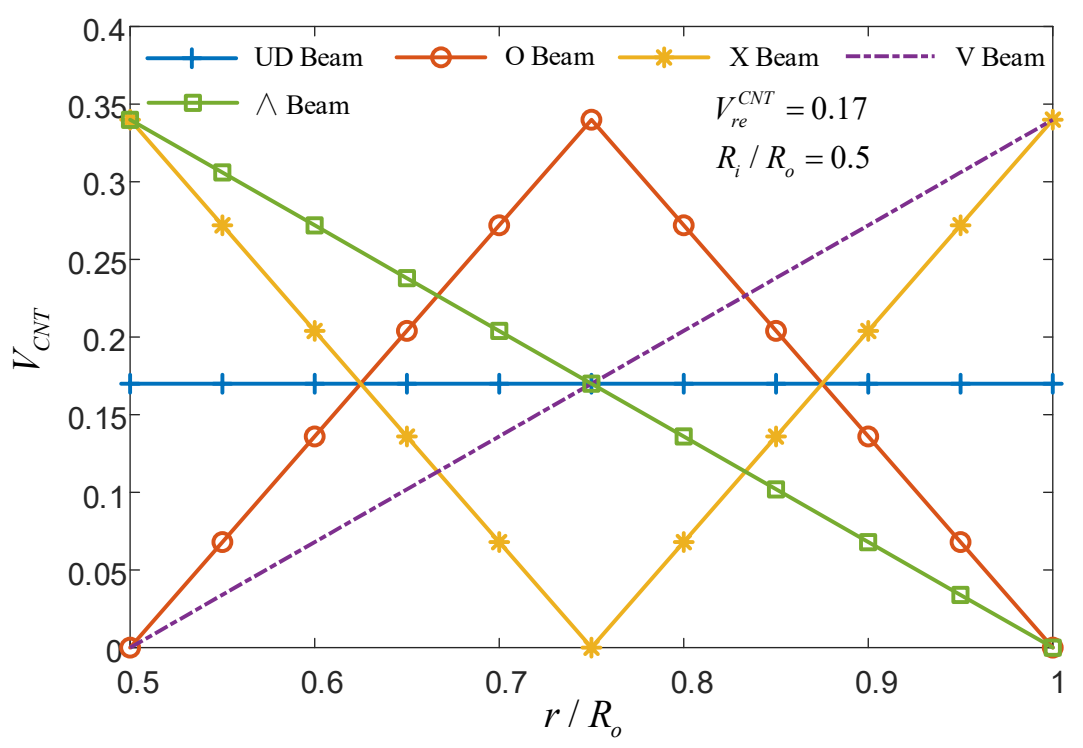

Fig2. Volume fractions of the carbon nanotube distribution along thickness for different FG-

$$
\text { CNTRC tube types }
$$

It can be seen that the UD-beam has uniformly distributed SWCNT reinforcement. By using the same rule, the thermal expansion coefficients have the form:

$$
\begin{aligned}
& \alpha_{11}(r)=\frac{\alpha_{11}^{C N T} V^{C N T} E_{11}^{C N T}+\alpha^{m} V^{m} E^{m}}{V^{C N T} E_{11}^{C N T}+V^{m} E^{m}} \\
& \alpha_{22}(r)=\left[\begin{array}{l}
\left(1+v_{12}^{C N T}\right) V^{C N T} \alpha_{22}^{C N T} \\
+\left(1+v^{m}\right) V^{m} \alpha^{m}-v_{12} \alpha_{11}
\end{array}\right]
\end{aligned}
$$

With $\alpha_{12}^{C N T}, \alpha_{22}^{C N T}, \alpha^{m}$ being thermal expansion coefficients of CNT and matrix, respectively.

We select $(10,10)$ SWCNT as reinforcement agents, and Poly-methyl methacrylate (PMMA) for matrix. the material properties $\rho^{C N T}, v_{12}^{C N T}$ and $\rho^{m}, v^{m}$ are assumed to depend weakly on the temperature variation. The CNT Efficiency parameters $\eta_{1}$ and $\eta_{2}$ are obtained by matching the Young's modulus of CNTRC obtained by the rule of mixture to those from the MD simulations given by ${ }^{[40]}$. The mechanical properties of composite beams such as elastic modulus and thermal expansion coefficient) change significantly at high temperatures, so it is necessary to consider the temperature dependence of materials in order to accurately predict the behavior of FG-CNTRC tube. The Eqs.1112 shows the relationship between the elastic modulus and thermal expansion coefficient of PMMA matrix and temperature.

$$
\alpha^{m}=45 \times[1+0.0005(T-300)] \times 10^{-6} / K
$$




$$
E^{m}=(3.52-0.0034 T) G P a
$$

Table 1 shows the material properties of $(10,10)$ SWCNT at different temperatures. The relationship is nonlinear. The CNT material properties (such as Young's modulus, shear modulus, thermal expansion coefficients are expressed as a continuous function of temperature by using polynomial fitting of 4 th order,

$$
P^{m}(T)=P_{0}+P_{1} \cdot T+P_{2} \cdot T^{2}+P_{3} \cdot T^{3}+P_{4} \cdot T^{4}
$$

Table 1 Temperature-dependent material properties of $(10,10)$ SWCNT $\left({ }^{[13]},{ }^{[41]}\right)$. Tube length $=$ $9.26 \mathrm{~nm}$, tube mean radius $=0.68 \mathrm{~nm}$, tube thickness $=0.067 \mathrm{~nm}$

\begin{tabular}{|c|c|c|c|c|c|c|c|}
\hline $\begin{array}{c}\text { Temperature } \\
(\mathrm{K})\end{array}$ & $\begin{array}{c}E_{11}^{C N T} \\
(\mathrm{GPa})\end{array}$ & $\begin{array}{c}E_{22}^{C N T} \\
(\mathrm{GPa})\end{array}$ & $\begin{array}{c}G_{12}^{C N T} \\
(\mathrm{GPa})\end{array}$ & $\begin{array}{c}\alpha_{11}^{C N T} \\
\left(\mathrm{x} 10^{-6} / \mathrm{K}\right)\end{array}$ & $\begin{array}{c}\alpha_{22}^{C N T} \\
\left(\mathrm{x} 10^{-6} / \mathrm{K}\right)\end{array}$ & $\begin{array}{c}\rho^{C N T} \\
\left(\mathrm{Kg} / \mathrm{m}^{3}\right)\end{array}$ & $v_{12}^{C N T}(-)$ \\
\hline 300 & 5646.6 & 7080 & 1944.5 & 3.4584 & 5.1682 & 1400 & 0.175 \\
\hline 400 & 5567.9 & 6981.4 & 1970.3 & 4.1496 & 5.0905 & 1400 & 0.175 \\
\hline 500 & 5530.8 & 6934.8 & 1964.3 & 4.5361 & 5.0189 & 1400 & 0.175 \\
\hline 700 & 5474.4 & 6864.1 & 1964.4 & 4.6677 & 4.8943 & 1400 & 0.175 \\
\hline 1000 & 5281.4 & 6622 & 1945.1 & 4.280 & 4.7532 & 1400 & 0.175 \\
\hline
\end{tabular}

Table 2 Temperature-dependent properties of PMMA $\left({ }^{[13]},{ }^{[42]}\right)$

\begin{tabular}{|c|c|c|c|c|}
\hline Temperature $(\mathrm{K})$ & $E^{m}(\mathrm{GPa})$ & $\alpha^{m}\left(\mathrm{x} 10^{-6} / \mathrm{K}\right)$ & $\rho^{m}\left(\mathrm{Kg} / \mathrm{m}^{3}\right)$ & $v^{m}(-)$ \\
\hline 300 & 2.5 & 45 & 1190 & 0.3 \\
\hline 400 & 2.16 & 47.25 & 1190 & 0.3 \\
\hline 500 & 1.82 & 49.5 & 1190 & 0.3 \\
\hline
\end{tabular}

Table 3 Fourth order interpolation coefficients

\begin{tabular}{|c|c|c|c|c|c|}
\hline & $P_{0}$ & $P_{1}$ & $P_{2}$ & $P_{3}$ & $P_{4}$ \\
\hline$E_{11}^{\mathrm{CNI}}(\mathrm{TPa})$ & 5.4744 & 5.5308 & 5.5679 & 5.6466 & 5.4744 \\
\hline$E_{22}^{\mathrm{CNI}}(\mathrm{TPa})$ & 6.8641 & 6.9348 & 6.9814 & 7.08 & 6.8641 \\
\hline$G_{12}^{\mathrm{CNI}}(\mathrm{TPa})$ & 1.9644 & 1.9643 & 1.9703 & 1.9445 & 1.9644 \\
\hline$\alpha_{11}^{C N T}\left(\mathrm{\times} 10^{-6} / \mathrm{K}\right)$ & 4.28 & 4.6677 & 4.5361 & 4.1496 & 3.4584 \\
\hline$\alpha_{22}^{C N T}\left(\mathrm{x} 10^{-6} / \mathrm{K}\right)$ & 4.7532 & 4.8943 & 5.0189 & 5.0905 & 5.1682 \\
\hline
\end{tabular}

Table 4 Efficiency parameters for PMMA/CNT $\left({ }^{[13]}\right)$

\begin{tabular}{|c|c|c|c|}
\hline$V_{r e}^{C N T}$ & $\eta_{1}$ & $\eta_{2}$ & $\eta_{3}$ \\
\hline 0.12 & 0.137 & 1.022 & 1.022 \\
\hline 0.17 & 0.142 & 1.626 & 1.626 \\
\hline 0.28 & 0.141 & 1.585 & 1.585 \\
\hline
\end{tabular}




\subsection{Governing equations}

Based on the higher-order shear deformation beam model for tubes ${ }^{[43]}$, the displacement field of the tubes is expressed as:

$$
\begin{aligned}
& u_{1}(x, y, z, t)=u(x, t)+f \cdot \frac{\partial w(x, t)}{\partial x}+g \bullet \varphi(x, t) \\
& u_{2}(x, y, z, t)=0 \\
& u_{3}(x, y, z, t)=w(x, t)
\end{aligned}
$$

Here, $u(x, t), w(x, t)$ are the longitudinal (x) and transverse $(\mathrm{z})$ displacement at the middle plane of the tube, and $\varphi_{i}(x, t)$ is the bending rotation of the cross-section, and

$$
f=\frac{z\left(3 R_{o}^{2} R_{i}^{2} r^{-2}-r^{2}\right)}{3\left(R_{o}^{2}+R_{i}^{2}\right)}, g=f+z, r^{2}=y^{2}+z^{2}
$$

It should be mentioned that, the position of neutral axis and centroid of the cross section is the same for present tubes. Based on the von Karman assumption, the nonlinear geometrical relationship of the tube can be expressed as:

$$
\begin{aligned}
& \varepsilon_{x x}=\frac{d u}{d x}+f \cdot \frac{d^{2} w}{d x^{2}}+g \cdot \frac{d \varphi}{d x}+\frac{1}{2}\left(\frac{d w}{d x}\right)^{2} \\
& \gamma_{x y}=\frac{\partial f}{\partial y}\left(\frac{d w}{d x}+\varphi\right) \\
& \gamma_{x z}=\frac{\partial g}{\partial z}\left(\frac{d w}{d x}+\varphi\right)
\end{aligned}
$$

The constitutive relationships of the tube considering a uniform temperature field are as follows:

$$
\begin{aligned}
& \sigma_{x x}=E_{11} \varepsilon_{x x}-E_{11} \alpha_{x} \Delta T \\
& \tau_{x y}=G_{12} \gamma_{x y} \\
& \tau_{x z}=G_{13} \gamma_{x z}
\end{aligned}
$$

Where $E_{11}, G_{12}, G_{13}$ are Young's modulus and shear modulus, $\alpha_{x}$ is the thermal expansion coefficient along $\mathrm{x}$ direction, and $\Delta T$ is the temperature offset from the reference temperature at which the tube in a stress free state.

The Hamilton's variational principle is implemented to derive the partial differential equation of the motion as follows: 


$$
\int_{t_{1}}^{t_{2}}\left(\delta U_{s}-\delta U_{k}+\delta U_{w}\right) d t=0
$$

The virtual strain energy of the tube $\delta U_{s}$ is given by

$$
\begin{aligned}
& \delta U_{s}=\delta \frac{1}{2} \int_{V}\left(\sigma_{x x} \varepsilon_{x x}+\tau_{x y} \gamma_{x y}+\tau_{x z} \gamma_{x z}\right) d V \\
& =\int_{0}^{L} \int_{A}\left(\sigma_{x x} \delta \varepsilon_{x x}+\tau_{x y} \delta \gamma_{x y}+\tau_{x z} \delta \gamma_{x z}\right) d A d x
\end{aligned}
$$

Substitute Eq.16 into Eq.19 results in the following relations:

$$
\delta U_{s}=\int_{0}^{L}\left(\begin{array}{l}
N_{x}\left(\frac{d \delta u}{d x}+\frac{d w}{d x} \frac{d \delta w}{d x}\right)+M_{x} \frac{d^{2} \delta w}{d x^{2}} \\
+P_{x} \frac{d \delta \varphi}{d x}+Q_{x r}\left(\frac{d \delta w}{d x}+\delta \varphi\right)
\end{array}\right) d x
$$

Where

$$
\left[N_{x}, M_{x}, P_{x}, Q_{x r}\right]=\int_{A}\left[\sigma_{x x}, \sigma_{x x} f, \sigma_{x x} g,\left(\tau_{x y} \frac{\partial f}{\partial y}+\tau_{x z} \frac{\partial g}{\partial z}\right)\right] d A
$$

The virtual kinetic energy of the fluid-conveying tube $\delta U_{k}$ is given by

$$
\delta U_{k}=\left[\begin{array}{l}
\int_{V}\left(\rho \frac{\partial u_{1}}{\partial t} \frac{\partial \delta u_{1}}{\partial t}+\rho \frac{\partial u_{3}}{\partial t} \frac{\partial \delta u_{3}}{\partial t}\right) d V \\
+\frac{1}{2} m_{f} \int_{0}^{L} \delta\left(v_{f}+\frac{\partial u}{\partial t}+\frac{\partial u}{\partial x} v_{f}\right)^{2} d x \\
+\frac{1}{2} m_{f} \int_{0}^{L} \delta\left(\frac{\partial \omega}{\partial t}+\frac{\partial \omega}{\partial x} v_{f}\right)^{2} d x
\end{array}\right]
$$

Substitute Eq.16 into Eq.22 results in the following relations:

$$
\begin{array}{r}
\delta U_{k}=\int_{0}^{L}\left[\begin{array}{l}
I_{0}\left(\frac{\partial u}{\partial t}\right) \delta \frac{\partial u}{\partial t}+I_{0} \frac{\partial w}{\partial t} \delta \frac{\partial w}{\partial t}+\left(I_{1} \frac{\partial^{2} w}{\partial x \partial t}+I_{2} \frac{\partial \varphi}{\partial t}\right) \cdot \delta \frac{\partial^{2} w}{\partial x \partial t} \\
+\left(I_{2} \frac{\partial^{2} w}{\partial x \partial t}+I_{3} \frac{\partial \varphi}{\partial t}\right) \cdot \delta \frac{\partial \varphi}{\partial t}
\end{array}\right] d x \\
+\int_{0}^{L}\left[\begin{array}{l}
I_{f 1} \delta \frac{\partial u}{\partial t}+I_{f 0} \frac{\partial u}{\partial t} \delta \frac{\partial u}{\partial t}+I_{f 1} \frac{\partial u}{\partial x} \delta \frac{\partial u}{\partial t}+N_{x}^{f} \delta \frac{\partial u}{\partial x}+I_{f 1} \frac{\partial u}{\partial t} \delta \frac{\partial u}{\partial x} \\
+N_{x}^{f} \frac{\partial u}{\partial x} \delta \frac{\partial u}{\partial x}+I_{f 0} \frac{\partial \omega}{\partial t} \delta \frac{\partial \omega}{\partial t}+I_{f 1} \frac{\partial \omega}{\partial x} \delta \frac{\partial \omega}{\partial t}+I_{f 1} \frac{\partial \omega}{\partial t} \delta \frac{\partial \omega}{\partial x} \\
+N_{x}^{f} \frac{\partial \omega}{\partial x} \delta \frac{\partial \omega}{\partial x}
\end{array}\right] d x
\end{array}
$$

With 


$$
\begin{gathered}
{\left[I_{0}, I_{1}, I_{2}, I_{3}\right]=\int_{A}\left[\rho, \rho f^{2}, \rho f g, \rho g^{2}\right] d A} \\
{\left[I_{f 0}, I_{f 1}, N_{x}^{f}\right]=\left[m_{f}, m_{f} v_{f}, m_{f} v_{f}^{2}\right]}
\end{gathered}
$$

Where

$m_{f}=\rho_{f} A, \rho_{f}$ is the density of the fluid, $v_{f}$ is the velocity of the flow and $A$ is the cross sectional of the flow.

The virtual work of the external pressure $\delta U_{w}$ is given by

$$
\delta U_{w}=-\int_{0}^{L} q \delta w d x
$$

Where $q$ is the load per unit length along $\mathrm{z}$ direction.

When the in-plane inertia is neglected, according to Hamiltonian variational principle, the governing equation represented by general force and moment is:

$$
\begin{aligned}
& -\frac{\partial N_{x}}{\partial x}=0 \\
& {\left[\begin{array}{l}
-\frac{\partial}{\partial x}\left(N_{x} \frac{\partial w}{\partial x}\right)+\frac{\partial^{2} M_{x}}{\partial x^{2}}-\frac{\partial Q_{x r}}{\partial x}+\left(I_{0}+I_{f 0}\right) \frac{\partial^{2} w}{\partial t^{2}} \\
-I_{1} \frac{\partial^{4} w}{\partial x^{2} \partial t^{2}}-I_{2} \frac{\partial^{3} \varphi}{\partial x \partial t^{2}}-q+2 I_{f 1} \frac{\partial^{2} \omega}{\partial x \partial t}+N_{x}^{f} \frac{\partial^{2} \omega}{\partial x^{2}}
\end{array}\right]=0} \\
& -\frac{\partial P_{x}}{\partial x}+Q_{x r}+I_{2} \frac{\partial^{3} w}{\partial x \partial t^{2}}+I_{3} \frac{\partial^{2} \varphi}{\partial t^{2}}=0
\end{aligned}
$$

From the first equation in Eq.27, we can find that the $N_{x}$ is a constant value. Due to the simply supported boundary conditions $(u(0)=u(L)=0)$, the expressing of $N_{x}$ can be given as,

$$
N_{x}=\frac{A_{1}}{2 L} \int_{0}^{L}\left(\frac{\partial \omega}{\partial x}\right)^{2} d x-N_{x}^{T}
$$

Substituting Eq.17 back into Eq.21 results in the following relations: 


$$
\begin{aligned}
& N_{x}=A_{1}\left(\frac{d u}{d x}+\frac{1}{2}\left(\frac{d w}{d x}\right)^{2}\right)-N_{x}^{T} \\
& M_{x}=A_{2} \frac{d^{2} w}{d x^{2}}+A_{3} \frac{d \varphi}{d x} \\
& P_{x}=A_{3} \frac{d^{2} w}{d x^{2}}+A_{4} \frac{d \varphi}{d x} \\
& Q_{x r}=A_{5}\left(\frac{d w}{d x}+\varphi\right)
\end{aligned}
$$

With

$$
\begin{aligned}
& {\left[A_{1}, A_{2}, A_{3}, A_{4}\right]=\int_{A}\left[E_{11}, E_{11} f^{2}, E_{11} f g, E_{11} g^{2},\right] d A} \\
& A_{5}=\int_{A}\left[G_{12}\left(\frac{\partial f}{\partial y}\right)^{2}+G_{13}\left(\frac{\partial g}{\partial z}\right)^{2}\right] d A \\
& N_{x}^{T}=\int_{A} E_{11} \alpha_{x} \Delta T d A
\end{aligned}
$$

Substituting Eq.30 into Eq.27, the governing equations represented by displacement components can be given as,

$$
\left[\begin{array}{l}
A_{2} \frac{d^{4} w}{d x^{4}}+A_{3} \frac{d^{3} \varphi}{d x^{3}}-A_{5}\left(\frac{d^{2} w}{d x^{2}}+\frac{d \varphi}{d x}\right)-\frac{A_{1}}{2 L} \int_{0}^{L}\left(\frac{\partial \omega}{\partial x}\right)^{2} d x \frac{\partial^{2} \omega}{\partial x^{2}} \\
+N_{x}^{T} \frac{\partial^{2} \omega}{\partial x^{2}}+\left(I_{0}+I_{f 0}\right) \frac{\partial^{2} \omega}{\partial t^{2}}-I_{1} \frac{\partial^{4} \omega}{\partial x^{2} \partial t^{2}}-I_{2} \frac{\partial^{3} \varphi}{\partial x \partial t^{2}} \\
+2 I_{f 1} \frac{\partial^{2} \omega}{\partial x \partial t}+N_{x}^{f} \frac{\partial^{2} \omega}{\partial x^{2}}
\end{array}\right]=q_{0}
$$

For generality, the following dimensionless parameters are introduced:

$$
\begin{aligned}
& \hat{x}=\frac{\pi x}{L}, \widehat{\omega}=\frac{\omega}{L}, \hat{\varphi}=\frac{\varphi}{\pi} \tau=\frac{\pi t}{L} \sqrt{\frac{E_{0}}{\rho_{0}}} \\
& \widehat{A_{1}}=\frac{L^{2}}{J \pi^{2}} A_{1} ; \widehat{A_{2}}=\frac{A_{2}}{J} ; \widehat{A_{3}}=\frac{A_{3}}{J} ; \widehat{A_{4}}=\frac{A_{4}}{J} ; \widehat{A_{5}}=\frac{L^{2}}{J \pi^{2}} A_{5} ; \widehat{N_{x}^{T}}=\frac{L^{2}}{J \pi^{2}} N_{x}^{T} \\
& \widehat{I_{0}}=\frac{L^{2}}{J \pi^{2}} \frac{E_{0}}{\rho_{0}} I_{0} ; \widehat{I_{1}}=\frac{E_{0}}{J \rho_{0}} I_{1} ; \widehat{I_{2}}=\frac{E_{0}}{J \rho_{0}} I_{2} ; \widehat{I_{3}}=\frac{E_{0}}{J \rho_{0}} I_{3} ; \widehat{q_{0}}=\frac{L^{3}}{J \pi^{4}} q_{0} \\
& \widehat{I_{f 0}}=\frac{L^{2}}{J \pi^{2}} \frac{E_{0}}{\rho_{0}} I_{f 0} ; \widehat{I_{f 1}}=\frac{L^{2}}{J \pi^{2}} \frac{E_{0}}{\rho_{0}} I_{f 1} ; \widehat{N_{x}^{f}}=\frac{L^{2}}{J \pi^{2}} N_{x}^{f} ; J=\int_{A} E_{0} \cdot z^{2} d A
\end{aligned}
$$

In which $E_{0}=2.5 \mathrm{GPa}, \rho_{0}=1150 \mathrm{Kg} / \mathrm{m}^{3}$ are the reference values of Young's 
modulus and mass density.

Considering the dimensionless parameters, the governing equations of the FGCNTRC fluid-conveying tube in thermal environment based on higher-order shear deformation beam theory are rewritten in the following form,

$$
\left[\begin{array}{l}
\widehat{A_{2}} \frac{\partial^{4} \hat{\omega}}{\partial \hat{x}^{4}}+\widehat{A}_{3} \frac{\partial^{3} \hat{\varphi}}{\partial \hat{x}^{3}}-\widehat{A_{5}}\left(\frac{\partial^{2} \widehat{\omega}}{\partial \hat{x}^{2}}+\frac{\partial \hat{\varphi}}{\partial \hat{x}}\right)-\widehat{A_{1}} \frac{\pi}{2} \int_{0}^{\pi}\left(\frac{\partial \hat{\omega}}{\partial \hat{x}}\right)^{2} d \hat{x} \cdot \frac{\partial^{2} \hat{\omega}}{\partial \hat{x}^{2}} \\
+\left(\widehat{N_{x}^{f}}+\widehat{N_{x}^{T}}\right) \frac{\partial^{2} \widehat{\omega}}{\partial \hat{x}^{2}}+\left(\widehat{I_{0}}+\widehat{I_{f 0}}\right) \frac{\partial^{2} \widehat{\omega}}{\partial \tau^{2}}-\widehat{I_{1}} \frac{\partial^{4} \widehat{\omega}}{\partial \hat{x}^{2} \partial \tau^{2}} \\
-\widehat{I_{2}} \frac{\partial^{3} \hat{\varphi}}{\partial \hat{x} \partial \tau^{2}}+2 \widehat{I_{f 1}} \frac{\partial^{2} \widehat{\omega}}{\partial \tau \partial \hat{x}}
\end{array}\right]=\widehat{q_{0}}
$$

One thing to be noted that the present higher-order shear deformation model can be degenerated to other beam models by choosing different shape functions f. For Euler beam, $f=-z$. For Timoshenko beam, $f=0$. For Reddy's beam, $f=-\frac{4 z^{3}}{3 h^{2}}$.

\subsection{Solving method}

A two-step perturbation technique ${ }^{[44]}$ is used to solve the nonlinear partial differential equations. In this case, the displacements and transverse load are expended as the following from,

$$
\begin{aligned}
& \hat{\omega}(\hat{x}, \tilde{t}, \varepsilon)=\sum_{i}^{n} \omega_{i}(\hat{x}, \tilde{t}) \cdot \varepsilon^{i} \\
& \hat{\varphi}(\hat{x}, \tilde{t}, \varepsilon)=\sum_{i}^{n} \varphi_{i}(\hat{x}, \tilde{t}) \cdot \varepsilon^{i} \\
& \widehat{q_{0}}(\hat{x}, \tilde{t}, \varepsilon)=\sum_{i}^{n} \lambda_{i}(\hat{x}, \tilde{t}) \cdot \varepsilon^{i}
\end{aligned}
$$

Here, the $\varepsilon$ is a small perturbation parameter with no physical meaning. It should be mentioned that, $\tilde{t}=\varepsilon \tau$ is introduced to delay the dynamic terms to higher-order perturbation equations.

Substituting Eq.34 into Eq.33, collecting the terms of $\varepsilon$ with the same order, we can obtain each order of perturbation equations, The first three order of perturbation equations are as follows, 


$$
\begin{aligned}
& O\left(\varepsilon^{1}\right):\left\{\begin{array}{l}
\widehat{A}_{2} \frac{\partial^{4} \omega_{1}}{\partial \hat{x}^{4}}+\widehat{A}_{3} \frac{\partial^{3} \varphi_{1}}{\partial \hat{x}^{3}}-\widehat{A_{5}}\left(\frac{\partial^{2} \omega_{1}}{\partial \hat{x}^{2}}+\frac{\partial \varphi_{1}}{\partial \hat{x}}\right)+\left(\widehat{N_{x}^{f}}+\widehat{N_{x}^{T}}\right) \frac{\partial^{2} \widehat{\omega}_{1}}{\partial \hat{x}^{2}}=\lambda_{1} \\
\widehat{A}_{3} \frac{\partial^{3} \omega_{1}}{\partial \hat{x}^{3}}+\widehat{A}_{4} \frac{\partial^{2} \varphi_{1}}{\partial \hat{x}^{2}}-\widehat{A_{5}}\left(\frac{\partial \omega_{1}}{\partial \hat{x}}+\varphi_{1}\right)=0
\end{array}\right. \\
& O\left(\varepsilon^{2}\right):\left\{\begin{array}{l}
\widehat{A_{2}} \frac{\partial^{4} \omega_{2}}{\partial \hat{x}^{4}}+\widehat{A}_{3} \frac{\partial^{3} \varphi_{2}}{\partial \hat{x}^{3}}-\widehat{A_{5}}\left(\frac{\partial^{2} \omega_{2}}{\partial \hat{x}^{2}}+\frac{\partial \varphi_{2}}{\partial \hat{x}}\right)+\left(\widehat{N_{x}^{f}}+\widehat{N_{x}^{T}}\right) \frac{\partial^{2} \omega_{2}}{\partial \hat{x}^{2}}=-2 \widehat{I_{f 1}} \frac{\partial^{2} \omega_{1}}{\partial \tilde{t} \partial \hat{x}}+\lambda_{2} \\
\widehat{A}_{3} \frac{\partial^{3} \omega_{2}}{\partial \hat{x}^{3}}+\widehat{A}_{4} \frac{\partial^{2} \varphi_{2}}{\partial \hat{x}^{2}}-\widehat{A_{5}}\left(\frac{\partial \omega_{2}}{\partial \hat{x}}+\varphi_{2}\right)=0
\end{array}\right.
\end{aligned}
$$

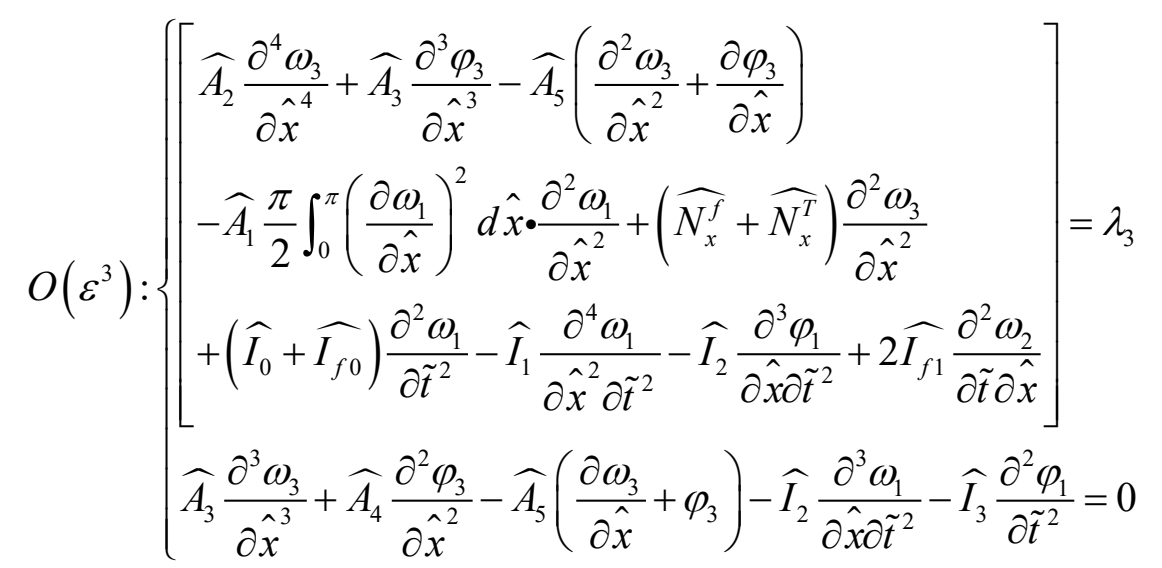

The solution of Eq.35 is assumed as follows,

$$
\omega_{1}(\hat{x}, \tilde{t})=A_{10}(\tilde{t}) \sin (m \hat{x}), \varphi_{1}(\hat{x}, \tilde{t})=B_{10}(\tilde{t}) \cos (m \hat{x}), m=1,2,3 \cdots
$$

Substituting Eq.38 into Eq.35 results in

$$
\begin{gathered}
B_{10}=\frac{-\widehat{A_{3}} m^{3}-\widehat{A}_{5} m}{\widehat{A}_{4} m^{2}+\widehat{A}_{5}} A_{10}=-\frac{\widehat{A}_{3} m^{3}+\widehat{A}_{5} m}{\widehat{A}_{4} m^{2}+\widehat{A}_{5}} A_{10} \\
\lambda_{1}=\left[\begin{array}{l}
\left.m^{4}\left[\widehat{A_{2}}-\widehat{A}_{3} \frac{\widehat{A}_{3} m^{2}+\widehat{A}_{5}}{\widehat{A}_{4} m^{2}+\widehat{A}_{5}}+\widehat{A_{5}}\left(\frac{\widehat{A_{4}}-\widehat{A_{3}}}{\widehat{A}_{4} m^{2}+\widehat{A}_{5}}\right)\right]\right] A_{10} \sin (m \hat{x}) \\
-\left(\widehat{N_{x}^{f}}+\widehat{N_{x}^{T}}\right) m^{2}
\end{array}\right]
\end{gathered}
$$

The solution of Eq.36 is assumed as follows

$$
\omega_{2}(\hat{x}, \tilde{t})=A_{20}(\tilde{t}) \sin (2 m \hat{x}), \varphi_{2}(\hat{x}, \tilde{t})=B_{20}(\tilde{t}) \cos (2 m \hat{x}), m=1,2,3 \cdots
$$

Substituting Eq.41 into Eq.36 results in 


$$
\begin{gathered}
A_{20}=\frac{\left(4 m^{2} \widehat{A}_{4}+\widehat{A}_{5}\right)}{\left(-8 m^{3} \widehat{A}_{3}-2 m \widehat{A}_{5}\right)} B_{20} \\
\lambda_{2}=\left[\begin{array}{l}
\left(16 m^{4} \widehat{A}_{2}+4 m^{2} \widehat{A}_{5}-4 m^{2}\left(\widehat{N_{x}^{f}}+\widehat{N_{x}^{T}}\right)\right) A_{20} \sin (2 m \hat{x}) \\
+\left(8 m^{3} \widehat{A}_{3}+2 m \widehat{A}_{5}\right) B_{20} \sin (2 m \hat{x})+2 \widehat{I_{f 1}} m \frac{\partial A_{10}}{\partial \hat{t}} \cos (m \hat{x})
\end{array}\right]
\end{gathered}
$$

The solution of Eq.37 is assumed as follows

$$
\omega_{3}(\hat{x}, \tilde{t})=A_{30}(\tilde{t}) \sin (3 m \hat{x}), \varphi_{3}(\hat{x}, \tilde{t})=B_{30}(\tilde{t}) \cos (m \hat{x}), m=1,2,3 \cdots
$$

Substituting Eq.44 into Eq.37 results in

$$
\begin{aligned}
& B_{30}=-\frac{1}{\left(\widehat{A}_{4} m^{2}+\widehat{A}_{5}\right)}\left(\widehat{I_{2}} m-\widehat{I}_{3} \frac{\widehat{A}_{3} m^{3}+\widehat{A}_{5} m}{\widehat{A}_{4} m^{2}+\widehat{A}_{5}}\right) \frac{\partial^{2} A_{10}}{\partial \tilde{t}^{2}} \\
& \lambda_{3}=\left[\begin{array}{l}
{\left[\widehat{A_{2}} 81 m^{4} A_{30}+\widehat{A}_{5} 9 m^{2} A_{30}-\left(\widehat{N_{x}^{f}}+\widehat{N_{x}^{T}}\right) 9 m^{2} A_{30}\right] \sin (3 m \hat{x})} \\
+\left[\left(\widehat{I_{0}}+\widehat{I_{f 0}}\right)+\widehat{I}_{1} m^{2}-2 \widehat{I_{2}} m \frac{\widehat{A_{3}} m^{3}+\widehat{A}_{5} m}{\widehat{A}_{4} m^{2}+\widehat{A}_{5}}+\widehat{I_{3}}\left(\frac{\widehat{A_{3}} m^{3}+\widehat{A}_{5} m}{\widehat{A}_{4} m^{2}+\widehat{A}_{5}}\right)^{2}\right] \frac{\partial^{2} A_{10}}{\partial \tilde{t}^{2}} \sin (m \hat{x}) \\
+2 m \frac{\partial A_{20}}{\partial \tilde{t}} \cos (2 m \hat{x})+\widehat{A}_{1} \frac{\pi^{2} m^{4}}{4}\left(A_{10}\right)^{3} \sin (m \hat{x})
\end{array}\right]
\end{aligned}
$$

Therefore, the final asymptotic solution is as follows

$$
\widehat{q_{0}}(\hat{x}, \tilde{t}, \varepsilon)=\lambda_{1}(\hat{x}, \tilde{t}) \cdot \varepsilon+\lambda_{2}(\hat{x}, \tilde{t}) \cdot \varepsilon^{2}+\lambda_{3}(\hat{x}, \tilde{t}) \cdot \varepsilon^{3}+O\left(\varepsilon^{4}\right)
$$

Since the nonlinear free vibration has no transverse load, i.e. $\widehat{q_{0}}=0$. we can obtain the following duffing equation based on Galerkin method,

$$
M \frac{\partial^{2}\left(\varepsilon A_{10}\right)}{\partial \tau^{2}}+K_{1}\left(\varepsilon A_{10}\right)+K_{3}\left(\varepsilon A_{10}\right)^{3}=0
$$

Where the $\tilde{t}$ has been replaced by $\tau$. 


$$
\begin{aligned}
& M=\left(\widehat{I_{0}}+\widehat{I_{f 0}}\right)+\widehat{I}_{1} m^{2}-2 \widehat{I_{2}} m \frac{\widehat{A_{3}} m^{3}+\widehat{A}_{5} m}{\widehat{A}_{4} m^{2}+\widehat{A}_{5}}+\widehat{I_{3}}\left(\frac{\widehat{A_{3}} m^{3}+\widehat{A}_{5} m}{\widehat{A}_{4} m^{2}+\widehat{A}_{5}}\right)^{2} \\
& K_{1}=m^{4}\left[\widehat{A_{2}}-\widehat{A_{3}} \frac{\widehat{A_{3}} m^{2}+\widehat{A}_{5}}{\widehat{A}_{4} m^{2}+\widehat{A}_{5}}+\widehat{A_{5}}\left(\frac{\widehat{A_{4}}-\widehat{A}_{3}}{\widehat{A}_{4} m^{2}+\widehat{A}_{5}}\right)\right]-\left(\widehat{N_{x}^{f}}+\widehat{N_{x}^{T}}\right) m^{2} \\
& K_{3}=\widehat{A}_{1} \frac{\pi^{2} m^{4}}{4}
\end{aligned}
$$

The closed-form solution of Eq can be expressed as,

$$
\omega_{N L}=\omega_{L} \sqrt{1+0.75 \frac{K_{3}}{K_{1}}\left(\frac{w_{\max }}{L}\right)^{2}}
$$

Where $\omega_{N L}$ and $\omega_{L}$ are the dimensionless nonlinear and linear frequencies and the form of $\omega_{L}$ is,

$$
\omega_{L}=\sqrt{\frac{K_{1}}{M}}
$$

According to Equation, the dimension nonlinear and linear frequencies, $\Omega_{N L}$ and $\Omega_{L}$, can be expressed as

$$
\Omega_{N L}=\omega_{N L} \frac{\pi}{L} \sqrt{\frac{E_{0}}{\rho_{0}}}
$$

\subsection{Results and discussions}

\subsubsection{Comparison studies}

To verify the accuracy of the theory in this paper, two comparison examples are provided in this section.

Table 5 gives the comparisons of dimensionless fundamental frequencies of FGM tube composed of metal and ceramics, with that in Ref. ${ }^{[45]}$. In this example, the tube is not supported on an elastic foundation. For detailed material parameters, please refer to the original paper. It can be clearly found that the results are highly consistent with the literature.

Table 5 Comparisons of dimensionless fundamental frequencies of FGM tube

\begin{tabular}{|l|l|l|l|l|l|l|}
\hline & 1 & 2 & 3 & 4 & 5 & 6 \\
\hline Ref. $^{[45]}$ & 0.011554 & 0.049112 & 0.106331 & 0.178023 & 0.25975 & 0.348242 \\
\hline Present & 0.011792 & 0.049329 & 0.106546 & 0.178239 & 0.25997 & 0.348468 \\
\hline
\end{tabular}


Fig.3 presents a comparison on dimension natural frequencies of an isotropic tube with parameters $L=10 m, R o=0.1 m, R i=0.08 m, E=210 G P a, \rho=7850 \mathrm{Kg} / \mathrm{m}^{3}$ with the results solved by commercial software. As seen from this figure, the results obtained by refined beam model are in good agreement with FEM results. The results using Euler beam model are larger at high frequency.

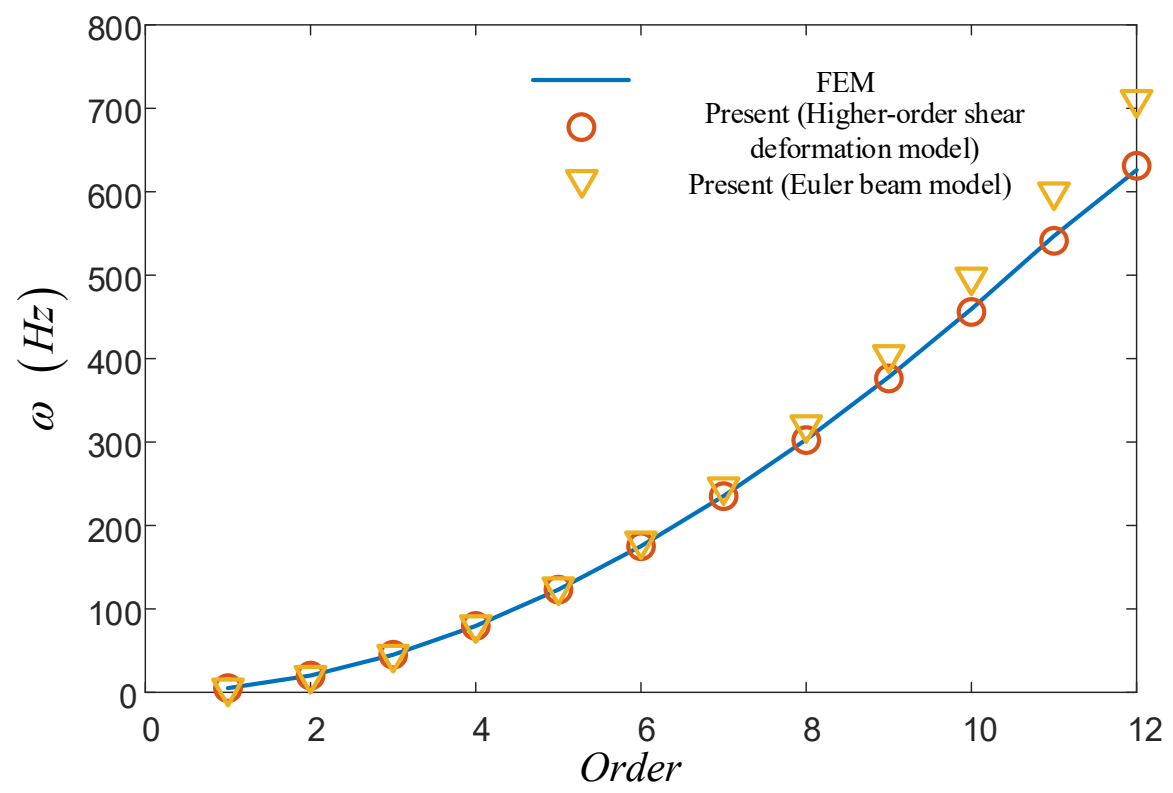

Fig. 3 Comparisons of dimension natural frequencies of isotropic tube

\subsubsection{Parameters studies}

\subsubsection{Vibration amplitude}

Fig.4 gives the nonlinear vibration frequency-amplitude curves of different patterns of reinforcement where $T=400 K, V_{r e}^{C N T}=0.17, R_{i}=0.5 R_{o}, L / R_{o}=30$, Matrix is PMMA, without fluid. It can be seen that, with the increase of amplitude, the nonlinear fundamental frequency $\left(\Omega_{N L}\right)$ and nonlinearity $\left(\omega_{N L} / \omega_{L}\right)$ both increase. Fig.4(a) displays that the nonlinearity of $\wedge$ beam is the most sensitive to amplitude change, followed by O-beam, UD-beam, X-beam, and V-beam. Fig.4(b) exhibits that, compared with large amplitude, the nonlinear fundamental frequency $\left(\Omega_{N L}\right)$ and nonlinearity $\left(\omega_{N L} / \omega_{L}\right)$ increase more slowly with the amplitude of vibration at small amplitude. With the increase of amplitude, the difference of nonlinear fundamental frequency with different patterns of reinforcement is almost unchanged. That is to say, the sensitivity of nonlinear frequency to amplitude change is the same.

Fig.5 present the nonlinear vibration frequency-amplitude curves of different volume fraction coefficients where $T=400 K, R_{i}=0.5 R_{o}, L / R_{o}=30$, Matrix is PMMA, without fluid, UD-beam. The results show that, with the increase of volume 
fraction coefficient, the nonlinearity $\left(\omega_{N L} / \omega_{L}\right)$ and nonlinear fundamental frequency $\left(\Omega_{N L}\right)$ are more sensitive to the amplitude change.

Fig.6 shows the nonlinear vibration frequency-amplitude curves of different temperatures where $V_{r e}^{C N T}=0.17, R_{i}=0.5 R_{o}, L / R_{o}=30$, Matrix is PMMA, without fluid, UD-beam. It can be found that, the nonlinearity is more sensitive to amplitude variation at high temperature. Compared with small amplitude, the difference of nonlinear fundamental frequency becomes smaller at large amplitude under different temperatures.

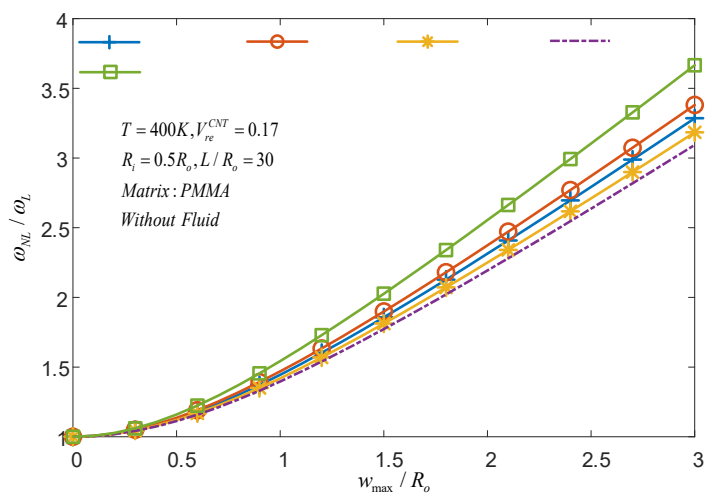

(a)

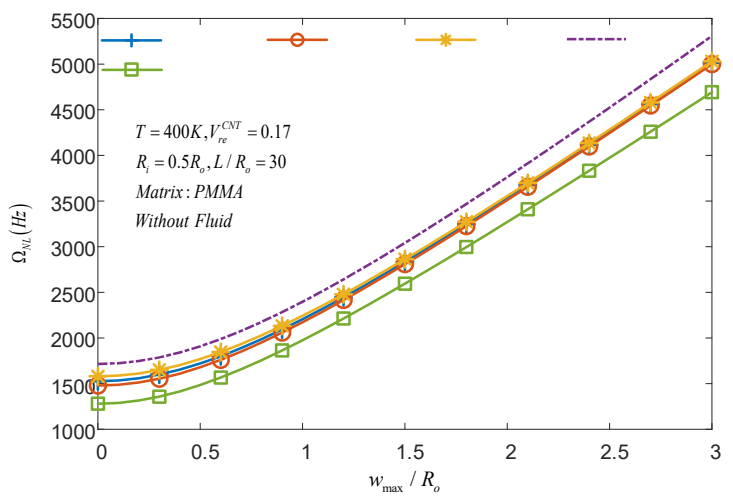

(b)

Fig. 4 Nonlinear vibration frequency-amplitude curves of different patterns of reinforcement.

(a) nonlinear-to-linear frequency ratio. (b) dimension nonlinear frequency

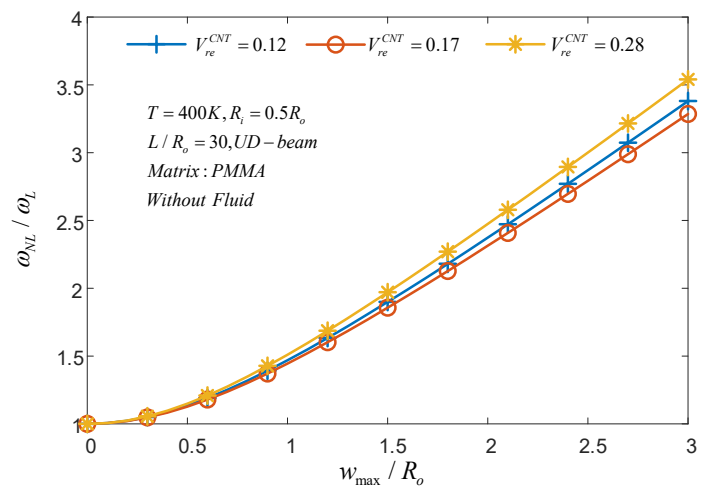

(a)

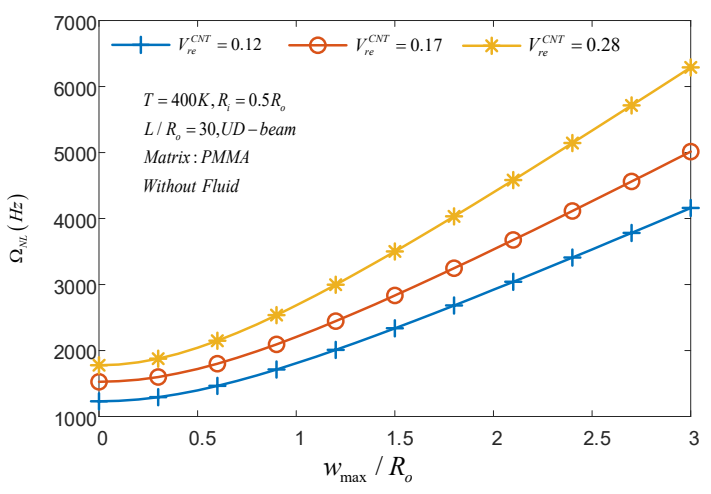

(b)

Fig. 5 Nonlinear vibration frequency-amplitude curves of different volume fraction coefficients. (a) nonlinear-to-linear frequency ratio. (b) dimension nonlinear frequency 


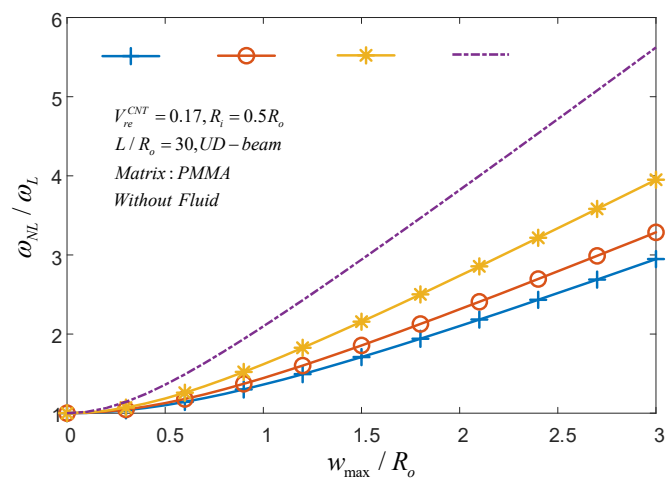

(a)

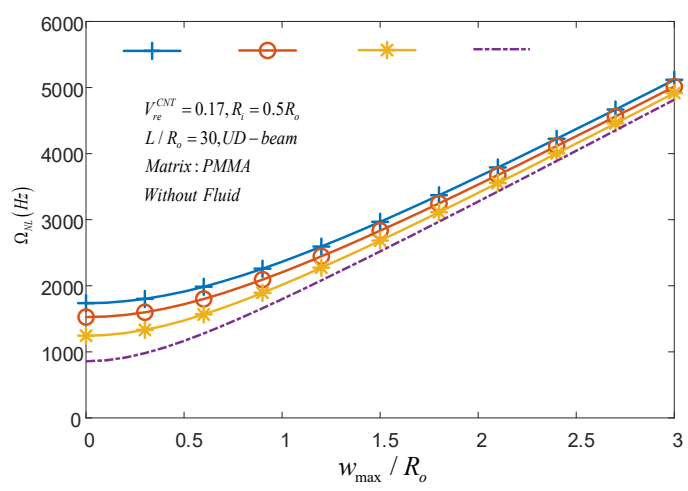

(b)

Fig. 6 Nonlinear vibration frequency-amplitude curves of different temperatures . (a) nonlinear-tolinear frequency ratio. (b) dimension nonlinear frequency

\subsubsection{Flow velocity}

The changes of nonlinear fundamental natural frequency $\left(\Omega_{N L}\right)$ with flow velocity of the FG-CNTRC tube for different patterns of reinforcement, volume fractions and geometrical ratio $R i / R o$ are shown in Fig.7-9.

It can be seen from Fig.7 that, the nonlinear fundamental frequency $\left(\Omega_{N L}\right)$ decreases as the flow velocity increases, while the change rate increases. A fundamental frequency of zero corresponds to a critical buckling flow velocity. V-beam has the highest critical buckling flow velocity, followed by X-beam, UD-beam, O-beam and $\wedge$-beam. Fig. 8 shows that the higher the volume fraction coefficient, the higher the critical buckling flow velocity of the tube. Fig. 9 shows that the critical buckling flow velocity increases as the ratio of inner diameter to outer diameter $(R i / R o)$ increases.

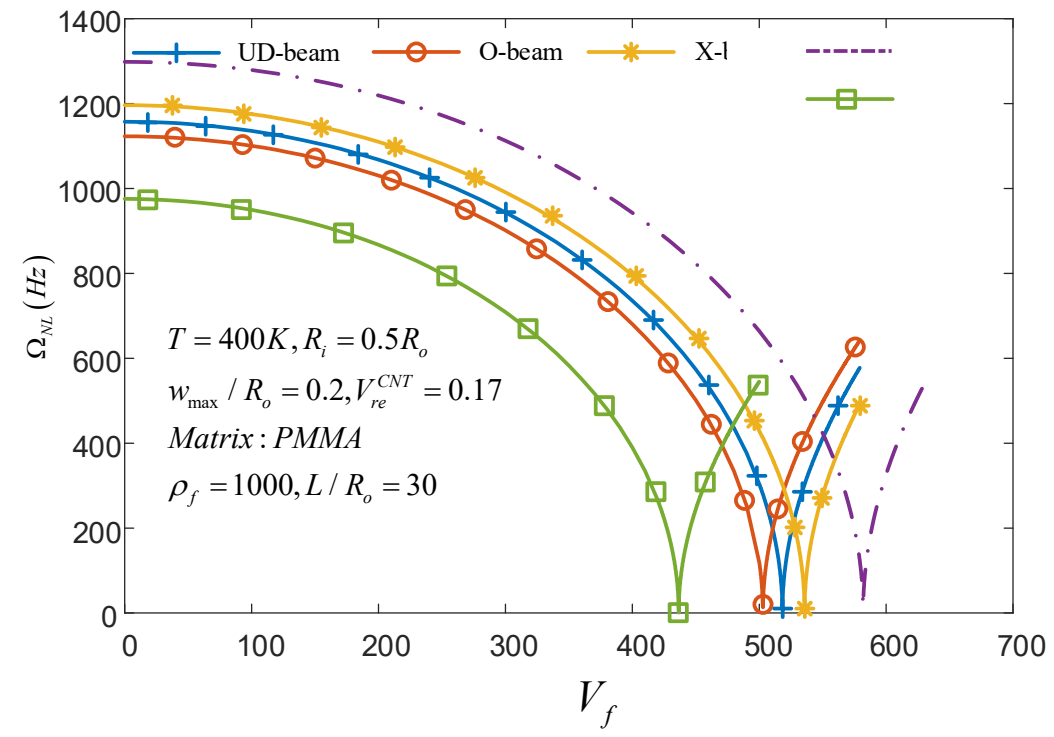

Fig. 7 Relationships between nonlinear frequency and flow velocity for various reinforced type 


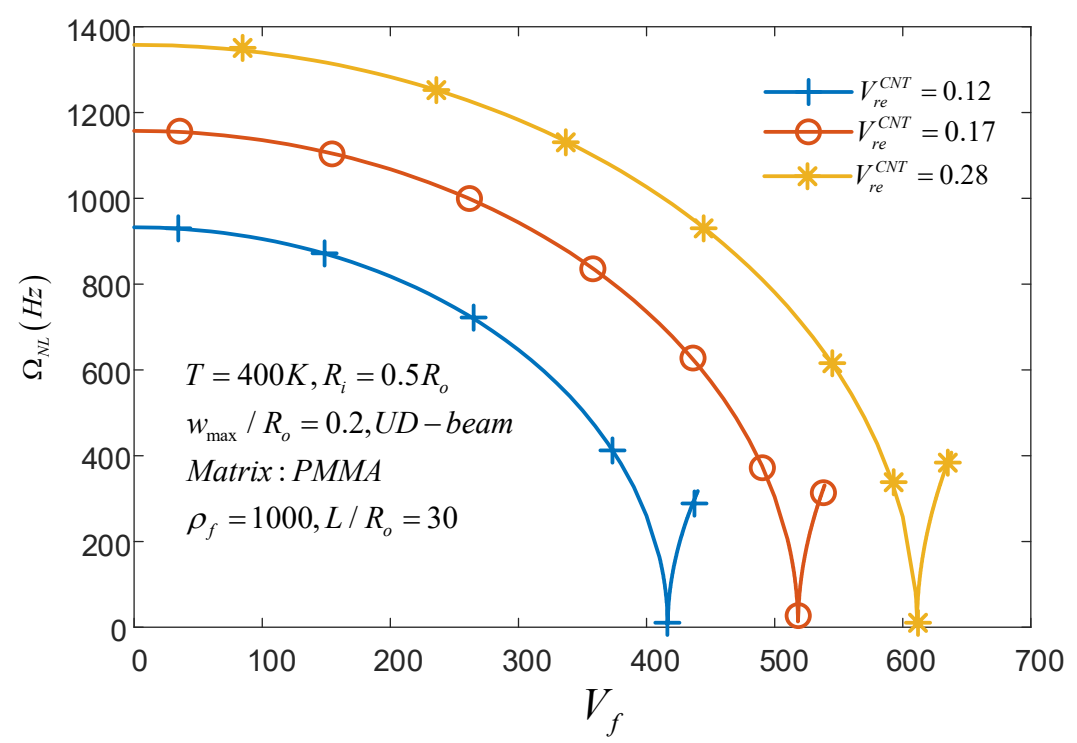

Fig. 8 Relationships between nonlinear frequency and flow velocity for various volume fraction coefficients

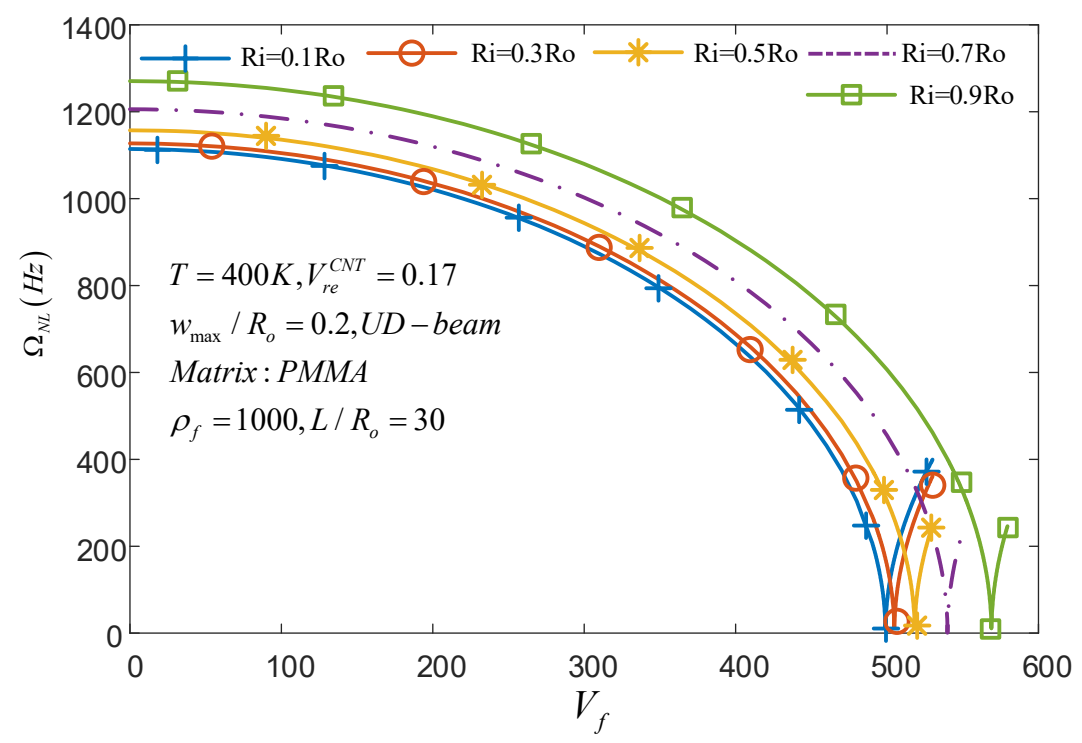

Fig. 9 Relationships between nonlinear frequency and flow velocity for various geometrical ratio $R i / R o$

\subsubsection{Temperature}

Fig.10-13 shows the relationships between nonlinear fundamental frequency and temperature for various patterns of reinforcement, volume fraction coefficients, geometrical ratio $L / R o$ and geometrical ratio $R i / R o$.

As can be seen from Fig.10, the nonlinear fundamental frequency decreases as the temperature increases. When the fundamental frequency is zero, it corresponds to the critical buckling temperature. Same as Fig.7, V beam has the highest critical buckling temperature among several patterns of reinforcement, followed by X beam, UD beam, 
$\mathrm{O}$ beam and $\wedge$ beam. Although the higher the volume fraction coefficient is, the higher the critical buckling flow velocity is, Fig.11 shows that the critical buckling temperature is the lowest when the volume fraction is 0.28 . Fig.12 gives that as the geometrical ratio $L / R o$ increases, the critical buckling temperature decreases, but the rate of change decreases. Fig. 13 shows that the critical buckling temperature increases with the increase of the ratio of inner to outer diameter $(R i / R o)$, but the change decreases when the ratio is small.

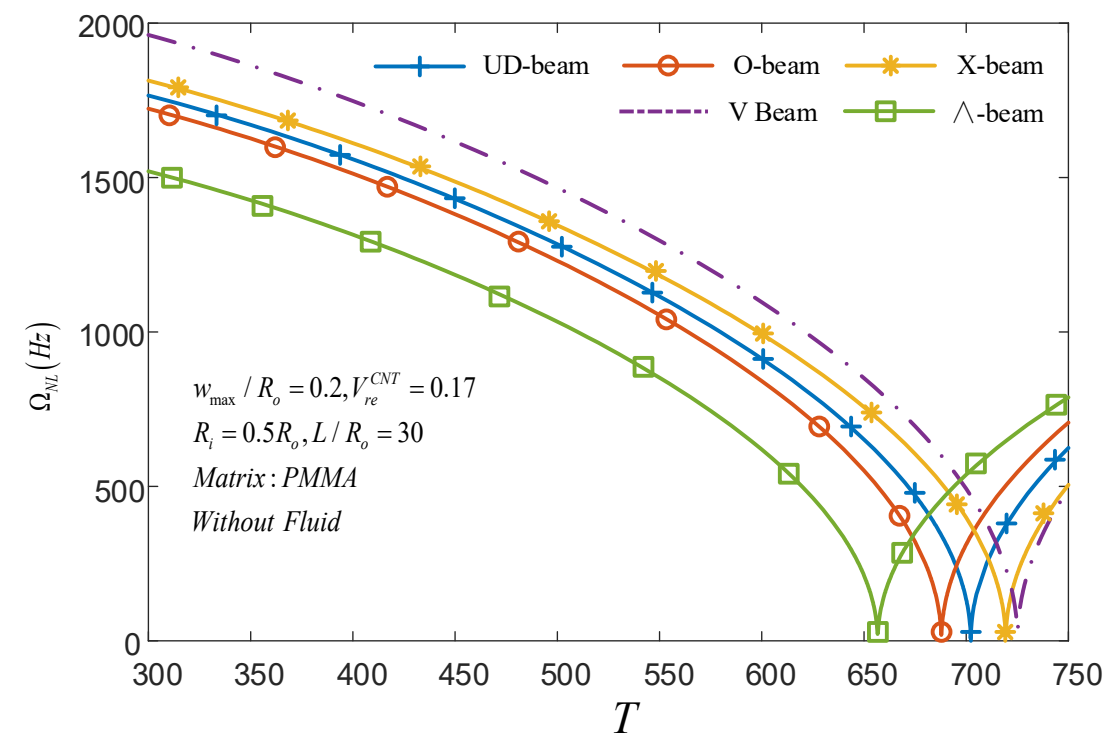

Fig. 10 Relationships between nonlinear frequency and temperature for various reinforced type

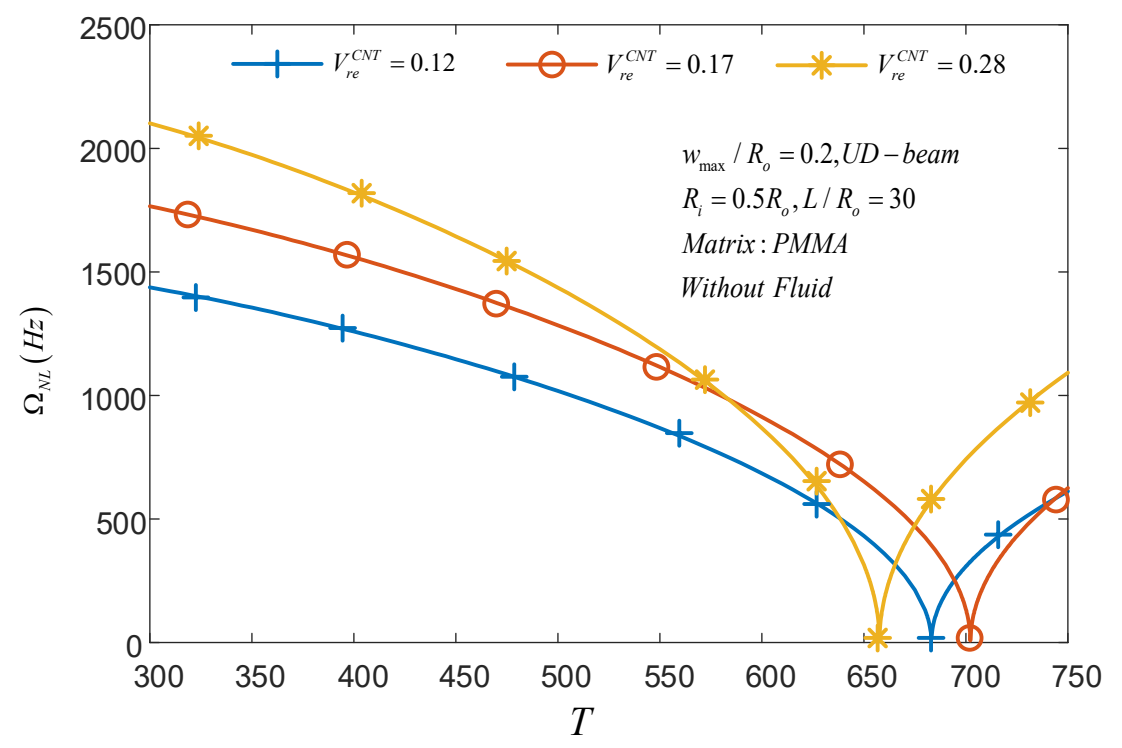

Fig. 11 Relationships between nonlinear frequency and temperature for various volume fraction 


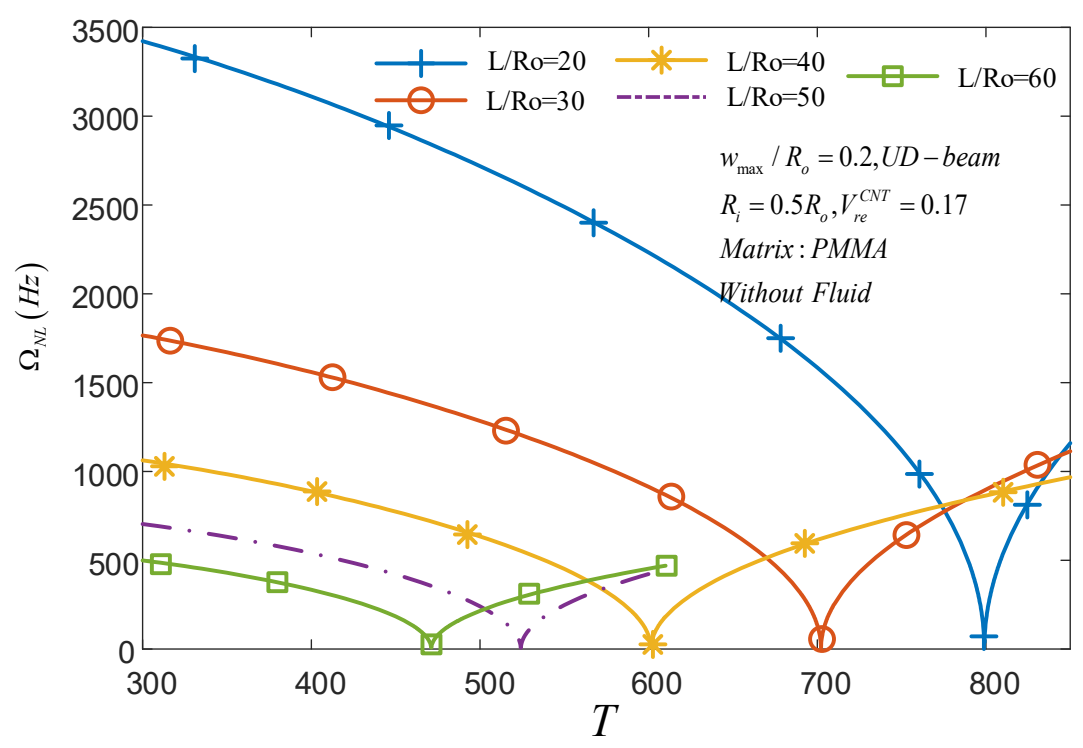

Fig. 12 Relationships between nonlinear frequency and temperature for various geometrical ratio $L / R o$

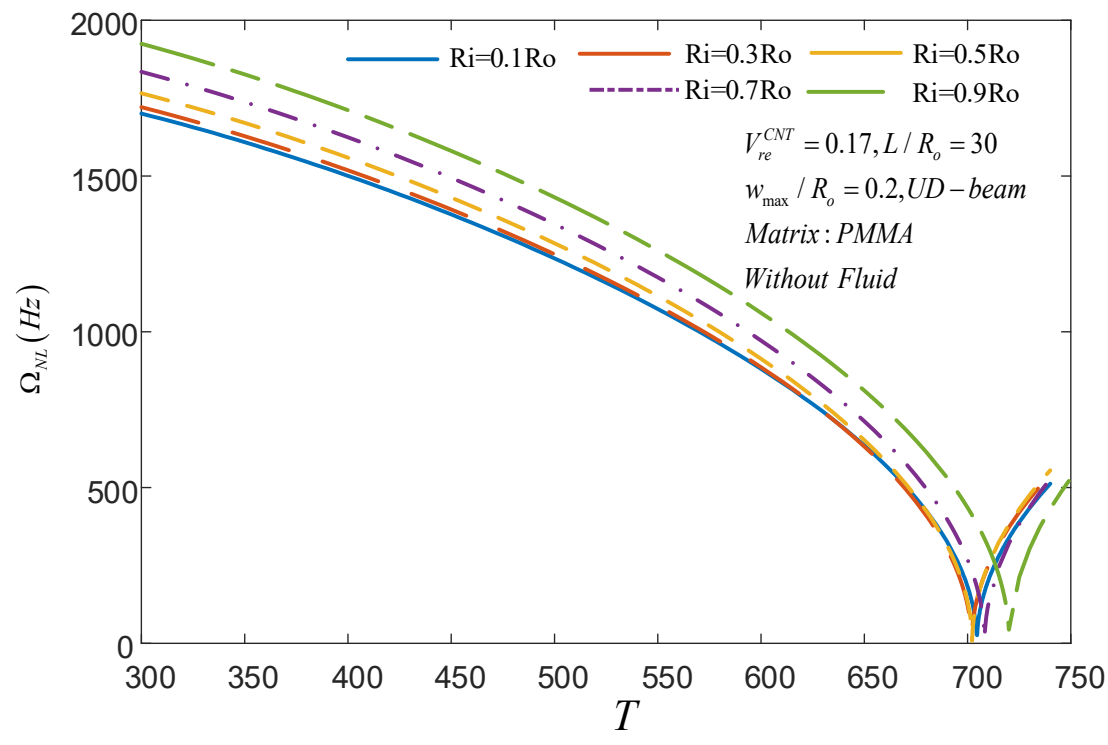

Fig. 13 Relationships between nonlinear frequency and temperature for various geometrical ratio $R i / R o$

Fig.14-15 show the influence of $L / R_{o}$ and $R_{i} / R_{o}$ on nonlinear fundamental frequency, respectively. It can be seen from Fig. 14 that the fundamental frequency decreases as the geometrical ratio $L / R_{o}$ increases, and when the fundamental frequency is zero, it corresponds to the critical buckling geometrical ratio. The nonlinearity varies greatly near the critical buckling geometrical ratio. Fig.15 shows that, with the $R_{i} / R_{o}$ increases, the nonlinear fundamental frequency increases. On the contrary, the nonlinearity decreases. 


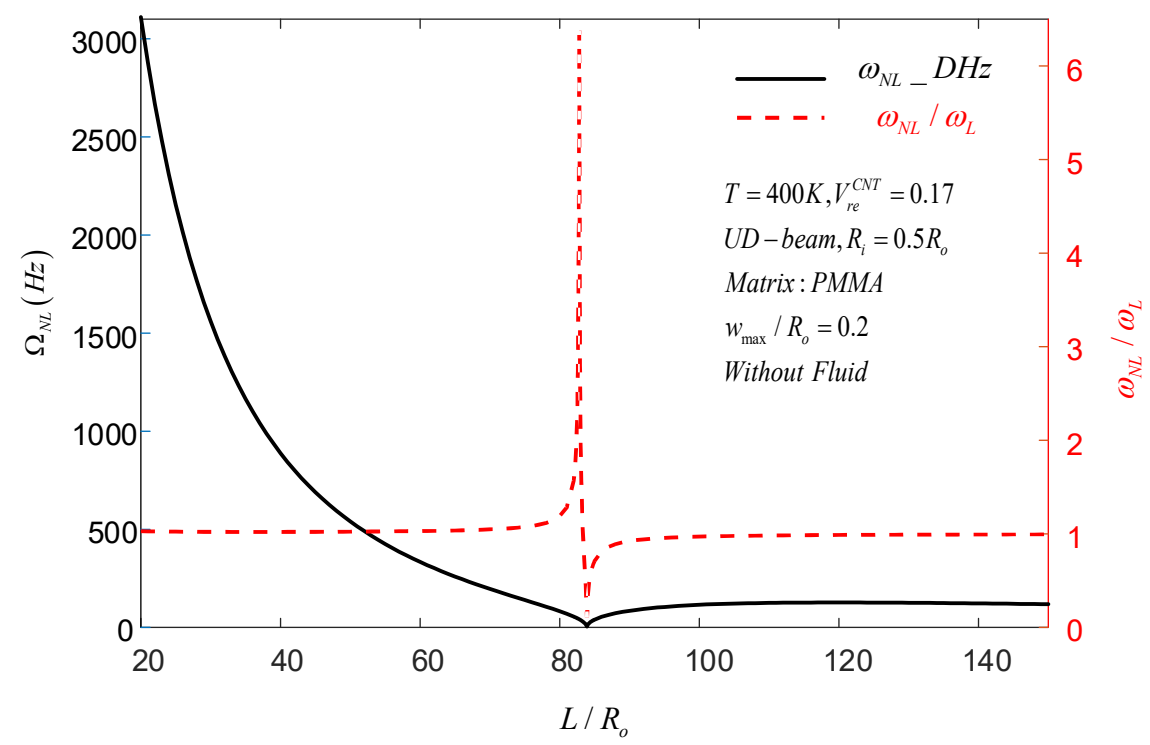

Fig. 14 Effect of parameter $L / R_{o}$ on natural frequency of FG-CNTRC tube

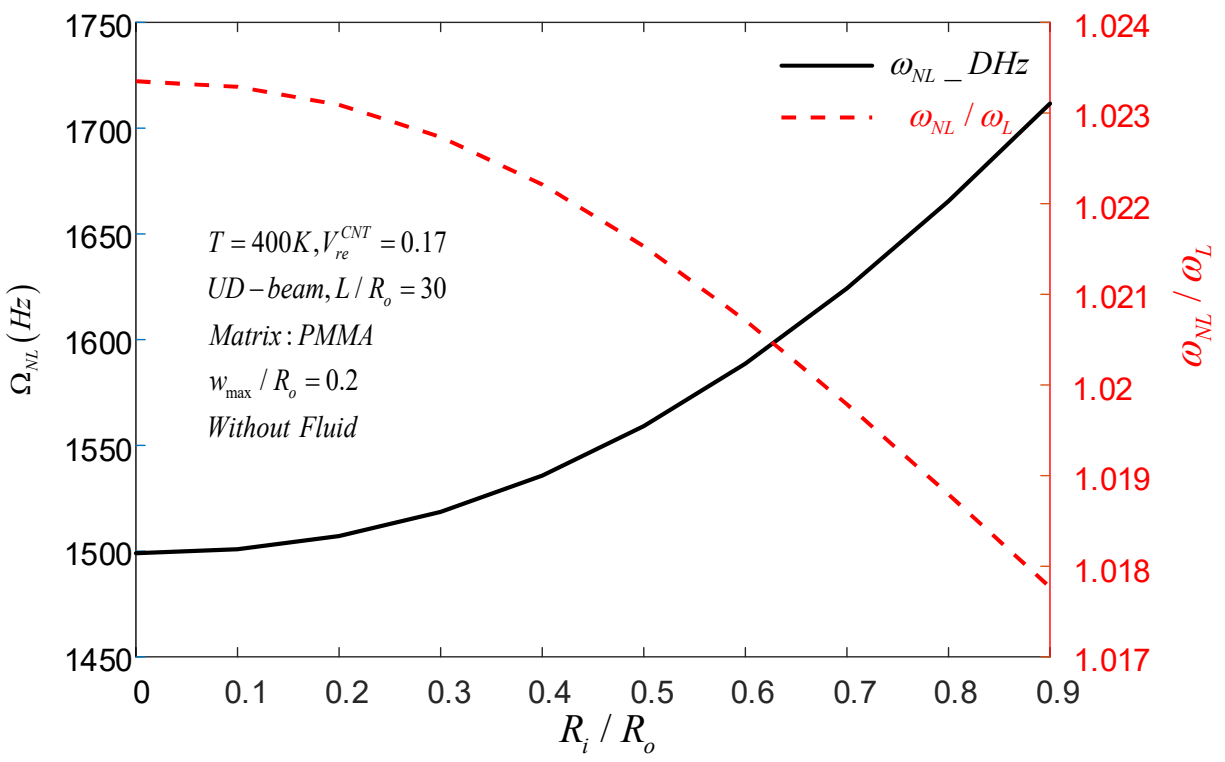

Fig. 15 Effect of parameter $R_{i} / R_{o}$ on natural frequency of FG-CNTRC tube

\subsection{Conclusion}

In this paper, an investigation on the nonlinear free vibration responses of functionally graded nanocomposite fluid-conveying tube reinforced by single-walled carbon nanotubes (SWNTs) in thermal environment is presented. A higher-order shear deformation theory and Hamilton's variational principle are employed to derive the motion equations incorporating the thermal and fluid effects. A two-step perturbation method is implemented to obtain the closed-form asymptotic solution for these 
nonlinear partial differential equations.

1) Compared with large amplitude, the nonlinear fundamental frequency $\left(\Omega_{N L}\right)$ and nonlinearity $\left(\omega_{N L} / \omega_{L}\right)$ increase more slowly with the amplitude of vibration at small amplitude.

2) V beam has the highest critical buckling flow velocity and temperature among several patterns of reinforcement, followed by X-beam, UD-beam, O-beam and $\wedge$-beam. However, the nonlinearity of $\wedge$-beam is the most sensitive to amplitude variation, followed by O-beam, UD-beam, X-beam, and V-beam.

3) Although the higher the volume fraction coefficient is, the higher the critical buckling flow velocity is, the critical buckling temperature is the lowest when the volume fraction is 0.28 . With the increase of volume fraction coefficient, the nonlinearity $\left(\omega_{N L} / \omega_{L}\right)$ and nonlinear fundamental frequency $\left(\Omega_{N L}\right)$ are more sensitive to the amplitude variation.

4) The nonlinearity $\left(\omega_{N L} / \omega_{L}\right)$ is more sensitive to amplitude variation at high temperature. Compared with small amplitude, the difference of nonlinear fundamental frequency $\left(\Omega_{N L}\right)$ becomes smaller at large amplitude under different temperatures.

5) The critical buckling flow velocity and temperature increases as the ratio of inner diameter to outer diameter $(R i / R o)$ increases, as does the nonlinear fundamental frequency $\left(\Omega_{N L}\right)$ increases. On the contrary, the nonlinearity decreases.

6) As the geometrical ratio $L / R o$ increases, the critical buckling temperature decreases, but the rate of change decreases.

\subsection{Declarations}

\subsubsection{Acknowledgments}

This work was supported by the National Natural Science Foundation of China under Grants 51575329, 61773254, 61625304 and 61873157; in part by Shanghai Rising-Star Program under Grants 17QA1401500; in part by Science and Technology Commission of Shanghai under Grants 16441909400 and 17DZ1205000.

\subsubsection{Conflict of Interest}

Conflict of Interest: The authors declare that they have no conflict of interest.

\subsubsection{Code availability}


Data will be made available on reasonable request.

\subsubsection{Authors' contribution}

All authors contributed to the study conception and design. Material preparation, data collection and analysis were performed by [Xu Chen], [Jing-Lei Zhao], [Gui-Lin She], [Yan Jing], [Hua-Yan Pu] and [Jun Luo]. The first draft of the manuscript was written by $[\mathrm{Xu} \mathrm{Chen}]$ and all authors commented on previous versions of the manuscript. All authors read and approved the final manuscript. 


\subsection{Reference}

[1] Yuan Yuan Zhang, Yu X. Wang, Xin Zhang, et al. On snap-buckling of FG-CNTR curved nanobeams considering surface effects[J]. Steel and Composite Structures, 2021, 38(3): 293304.

[2] Gui-Lin She, Hai-Bo Liu, Behrouz Karami. Resonance analysis of composite curved microbeams reinforced with graphene nanoplatelets[J]. Thin-Walled Structures, 2021, 160

[3] $\mathrm{Lu} \mathrm{Lu}$, Shuang Wang, Min Li, et al. Free vibration and dynamic stability of functionally graded composite microtubes reinforced with graphene platelets[J]. Composite Structures, 2021, 272

[4] Lu Lu, Gui-Lin She, Xingming Guo. Size-dependent postbuckling analysis of graphene reinforced composite microtubes with geometrical imperfection[J]. International Journal of Mechanical Sciences, 2021, 199

[5] O. Civalek, M. H. Jalaei. Shear buckling analysis of functionally graded (FG) carbon nanotube reinforced skew plates with different boundary conditions[J]. Aerospace Science and Technology, 2020, 99

[6] S. Iijima. HELICAL MICROTUBULES OF GRAPHITIC CARBON[J]. Nature, 1991, 354(6348): 56-58.

[7] S. Kanagaraj, Fátima R. Varanda, Tatiana V. Zhil’tsova, et al. Mechanical properties of high density polyethylene/carbon nanotube composites[J]. Composites Science and Technology, 2007, 67(15-16): 3071-3077.

[8] T. W. Odom, J. L. Huang, P. Kim, et al. Atomic structure and electronic properties of singlewalled carbon nanotubes[J]. Nature, 1998, 391(6662): 62-64.

[9] Kamyar Shirvanimoghaddam, Bhargav Polisetti, Aravind Dasari, et al. Thermomechanical performance of cheetah skin carbon nanotube embedded composite: Isothermal and nonisothermal investigation[J]. Polymer, 2018, 145: 294-309.

[10] Hui-Shen Shen. Nonlinear bending of functionally graded carbon nanotube-reinforced composite plates in thermal environments[J]. Composite Structures, 2009, 91(1): 9-19.

[11] - Torsional postbuckling of nanotube-reinforced composite cylindrical shells in thermal environments[J]. Composite Structures, 2014, 116: 477-488.

[12] Hui-Shen Shen, Y. Xiang. Nonlinear analysis of nanotube-reinforced composite beams resting on elastic foundations in thermal environments[J]. Engineering Structures, 2013, 56: 698-708.

[13] Hui-Shen Shen, Chen-Li Zhang. Thermal buckling and postbuckling behavior of functionally graded carbon nanotube-reinforced composite plates[J]. Materials \& Design, 2010, 31(7): 3403-3411.

[14] Nguyen Dinh Duc, Phuc Pham Minh. Free vibration analysis of cracked FG CNTRC plates using phase field theory[J]. Aerospace Science and Technology, 2021, 112

[15] Ali Ghorbanpour Arani, Farhad Kiani, Hassan Afshari. Free and forced vibration analysis of laminated functionally graded CNT-reinforced composite cylindrical panels[J]. Journal of Sandwich Structures \& Materials, 2021, 23(1): 255-278.

[16] M. Vinyas, D. Harursampath. Nonlinear vibrations of magneto-electro-elastic doubly curved shells reinforced with carbon nanotubes[J]. Composite Structures, 2020, 253

[17] A. H. Sofiyev, F. Tornabene, R. Dimitri, et al. Buckling Behavior of FG-CNT Reinforced Composite Conical Shells Subjected to a Combined Loading[J]. Nanomaterials (Basel), 2020, 
10(3)

[18] M. Rezaiee-Pajand, Emad Sobhani, Amir R. Masoodi. Free vibration analysis of functionally graded hybrid matrix/fiber nanocomposite conical shells using multiscale method[J]. Aerospace Science and Technology, 2020, 105

[19] Hanen Mallek, Hanen Jrad, Mondher Wali, et al. Dynamic analysis of functionally graded carbon nanotube-reinforced shell structures with piezoelectric layers under dynamic loads[J]. Journal of Vibration and Control, 2020, 26(13-14): 1157-1172.

[20] Farshad Khosravi, Mandi Simyari, Seyed A. Hosseini, et al. Size dependent axial free and forced vibration of carbon nanotube via different rod models[J]. Advances in Nano Research, 2020, 9(3): 157-172.

[21] Tao Fu, Zhaobo Chen, Hongying Yu, et al. Vibratory response and acoustic radiation behavior of laminated functionally graded composite plates in thermal environments[J]. Journal of Sandwich Structures \& Materials, 2020, 22(5): 1681-1706.

[22] D. S. Craveiro, M. A. R. Loja. A Study on the Effect of Carbon Nanotubes' Distribution and Agglomeration in the Free Vibration of Nanocomposite Plates[J]. C, 2020, 6(4)

[23] Hao Cheng, Chao-feng Li, Yulin Jiang. Free vibration analysis of rotating pre-twisted ceramic matrix carbon nanotubes reinforced blades[J]. Mechanics of Advanced Materials and Structures, 2020: 1-75.

[24] Yan Cao, Farayi Musharavati, Shahrizan Baharom, et al. Vibration response of FG-CNTreinforced plates covered by magnetic layer utilizing numerical solution[J]. Steel and Composite Structures, 2020, 37(2): 253-258.

[25] Noureddine Bendenia, Mohamed Zidour, Abdelmoumen Anis Bousahla, et al. Deflections, stresses and free vibration studies of FG-CNT reinforced sandwich plates resting on Pasternak elastic foundation[J]. Computers and Concrete, 2020, 26(3): 213-226.

[26] Mahsa Heidari, Hadi Arvin. Nonlinear free vibration analysis of functionally graded rotating composite Timoshenko beams reinforced by carbon nanotubes[J]. Journal of Vibration and Control, 2019, 25(14): 2063-2078.

[27] K. M. Liew, Z. X. Lei, L. W. Zhang. Mechanical analysis of functionally graded carbon nanotube reinforced composites: A review[J]. Composite Structures, 2015, 120: 90-97.

[28] Yaxin Zhen, Yafei Gong, Ye Tang. Nonlinear vibration analysis of a supercritical fluidconveying pipe made of functionally graded material with initial curvature[J]. Composite Structures, 2021, 268

[29] Feng Liang, An Gao, Xue-Feng Li, et al. Nonlinear parametric vibration of spinning pipes conveying fluid with varying spinning speed and flow velocity[J]. Applied Mathematical Modelling, 2021, 95: 320-338.

[30] Rahim Abdollahi, Rouhollah Dehghani Firouz-abadi, Mohammad Rahmanian. On the stability of rotating pipes conveying fluid in annular liquid medium[J]. Journal of Sound and Vibration, 2021, 494

[31] Bo Zhu, Qi Xu, Ming Li, et al. Nonlinear free and forced vibrations of porous functionally graded pipes conveying fluid and resting on nonlinear elastic foundation[J]. Composite Structures, 2020, 252

[32] K. Zhou, Q. Ni, L. Wang, et al. Planar and non-planar vibrations of a fluid-conveying cantilevered pipe subjected to axial base excitation[J]. Nonlinear Dynamics, 2020, 99(4): 2527-2549. 
[33] Xia Tan, Hu Ding, Jian-Qiao Sun, et al. Primary and super-harmonic resonances of Timoshenko pipes conveying high-speed fluid[J]. Ocean Engineering, 2020, 203

[34] Pooriya Shahali, Hassan Haddadpour, Seyed Ali Hosseini Kordkheili. Nonlinear dynamics of viscoelastic pipes conveying fluid placed within a uniform external cross flow[J]. Applied Ocean Research, 2020, 94

[35] A. R. Askarian, M. R. Permoon, M. Shakouri. Vibration analysis of pipes conveying fluid resting on a fractional Kelvin-Voigt viscoelastic foundation with general boundary conditions[J]. International Journal of Mechanical Sciences, 2020, 179

[36] Ze-Qi Lu, Kai-Kai Zhang, Hu Ding, et al. Nonlinear vibration effects on the fatigue life of fluid-conveying pipes composed of axially functionally graded materials[J]. Nonlinear Dynamics, 2020, 100(2): 1091-1104.

[37] Rasoul Khodabakhsh, Ali Reza Saidi, Reza Bahaadini. An analytical solution for nonlinear vibration and post-buckling of functionally graded pipes conveying fluid considering the rotary inertia and shear deformation effects[J]. Applied Ocean Research, 2020, 101

[38] M. Heshmati. Influence of an eccentricity imperfection on the stability and vibration behavior of fluid-conveying functionally graded pipes[J]. Ocean Engineering, 2020, 203

[39] Amal M. K. Esawi, Mahmoud M. Farag. Carbon nanotube reinforced composites: Potential and current challenges[J]. Materials \& Design, 2007, 28(9): 2394-2401.

[40] Yue Han, James Elliott. Molecular dynamics simulations of the elastic properties of polymer/carbon nanotube composites[J]. Computational Materials Science, 2007, 39(2): 315323.

[41] Jian Yang, Xu-Hao Huang, Hui-Shen Shen. Nonlinear Vibration of Temperature-Dependent FG-CNTRC Laminated Beams with Negative Poisson's Ratio[J]. International Journal of Structural Stability and Dynamics, 2020, 20(04)

[42] Nuttawit Wattanasakulpong, Variddhi Ungbhakorn. Analytical solutions for bending, buckling and vibration responses of carbon nanotube-reinforced composite beams resting on elastic foundation[J]. Computational Materials Science, 2013, 71: 201-208.

[43] $\mathrm{Pu}$ Zhang, Yiming Fu. A higher-order beam model for tubes[J]. European Journal of Mechanics - A/Solids, 2013, 38: 12-19.

[44] Shen, Hui-Shen. A Two-Step Perturbation Method in Nonlinear Analysis of Beams, Plates and Shells[M].Higher Education Press, 2013.

[45] Jun Zhong, Yiming Fu, Detao Wan, et al. Nonlinear bending and vibration of functionally graded tubes resting on elastic foundations in thermal environment based on a refined beam model[J]. Applied Mathematical Modelling, 2016, 40(17-18): 7601-7614. 